\title{
MODELLING THE AMOUNT OF CARBON STOCK USING REMOTE SENSING IN URBAN FOREST AND ITS RELATIONSHIP WITH LAND USE CHANGE
}

\author{
N.Tavasoli ${ }^{1, *}$, H.Arefi ${ }^{1}$, S.Samiei-Esfahany ${ }^{1}$, Q.Ronoud ${ }^{2}$ \\ ${ }^{1}$ School of Surveying and Geospatial Engineering, University of Tehran, Tehran, Iran- \\ (negar.tavasoli, hossein.arefi, s.samieiesfahany)@ ut.ac.ir \\ ${ }^{2}$ Faculty of Natural Resources, University of Tehran, Karaj, Iran- ronoud.q@ut.ac.ir
}

KEY WORDS: Above ground biomass, Carbon stock, Urban area, SVM-genetic, Satellite data

\begin{abstract}
:
The estimation of biomass has been highly regarded for assessing carbon sources. In this paper, ALOS PALSAR, Sentinel-1, Sentinel-2 and ground data are used for estimating of above ground biomass (AGB) with SVM-genetic model Moreover Landsat satellite data was used to estimate land use change detection. The wide range of vegetation, textural and principal component analysis (PCA) indices (using optical images) and backscatter, decomposition and textural features (from radar images) are derived together with in situ collected AGB data into model to predict AGB. The results indicated that the coefficient of determination (R2) for ALOS PALSAR, Sentinel-1, Sentinel-2 were $0.51,0.50$ and 0.60 respectively. The best accuracy for combining all data was 0.83. Afterwards, the carbon stock map was calculated. Landsat series data were acquired to document the spatiotemporal dynamics of green spaces in the study area. By using a supervised classification algorithm, multi-temporal land use/cover data were extracted from a set of satellite images and the carbon stock time series simulated by using carbon stock maps and green space (urban forest) maps.
\end{abstract}

\section{INTRODUCTION}

Carbon storage plays an important role in urban ecosystems. However, urban development leads to changes in urban ecosystems and hence menaces carbon storage. More than half of the global population now inhabits in urban areas, and this process will continue to increase at a rate of $4 \%$ a per decade by 2050 (UN 2015). Carbon management is one of the most effective climate change activities at national and international level. Decrease and increase Carbon emissions as Effective carbon management approaches have been proposed to reduce the rising rate of air temperature. Urban trees affect local climate, carbon cycle and Climate change energy consumption. Urban green space especially trees provide many benefits that can improve the quality of the environment and human health in urban places. These benefits include improving weather quality, energy conservation, air temperature reduction, ultraviolet radiation and other environmental and social benefits. Although, those benefits are needed high potential costs (Pataki, Carreiro et al. 2011). Thus, more studies are needed to accurately assess each ecosystem service provided by urban forests and clearly communicate the scientific findings. Cities account for $\% 75$ of global anthropogenic carbon dioxide (CO2) emissions (Seto, Dhakal et al. 2014). Remote sensing combined with ground data and modelling has developed technique to estimate carbon (C) storage and sequestration by urban forests (Rao, Hutyra et al. 2013). But information about carbon storage in urban ecosystems is still rare and more research is required. Estimation of the carbon storage by trees in urban environments would be advantageous for evaluating environmental and economic benefits of these types of ecosystems. The AGB Forest Estimation techniques based on remote sensing data have supported to extrapolate AGB measurements from field data over entire landscape (Saatchi, Harris et al. 2011). Remote sensing techniques cause to estimate of AGB at various scales. It is the main source for biomass estimation. Estimation and monitoring of carbon stock with Remote sensing data can be fast and low cost and it is also providing information from inaccessible locations that cannot be sampled (Kwak, Lee et al. 2010) Biomass estimation methods can be divided into two categories: parametric and non-parametric algorithms (Lu 2006). Choosing a good modelling approach is a challenging issue because the modelling approach is as important as the remote sensing data type in estimating carbon storage (Fassnacht, Hartig et al. 2014). In this study, the potential of Sentinel-1, ALOS PALSAR and Sentinel-2 imagery for the retrieval and predictive mapping of forests AGB estimation was evaluated. The specific objectives included the following issues: (1) to determine and model the relationship between fieldmeasured forests AGB and Sentinel-based predictors, including Sentinel-1 and ALOS PALSAR SAR backscatter information and Sentinel-2 multispectral indices based; (2) to evaluate and compare the accuracy of the biomass prediction models, including SVM-Genetic models; and (3) to map forest AGB spatial distribution by four optimal models.

Gaia Vaglio Laurin et al (2014) used lidar and hyperspectral data to estimate the AGB of an African tropical forest by using Multiplicative Power Model (MPM) and Partial Least Square Regression (PLSR) methods. PLSR had a better result than MPM. The result of integration of lidar and hyperspectral datawas best result that was $\mathrm{R} 2=0.70$ (Laurin, Chen et al. 2014).

Dirk Pflugmacher et al (2014) developed regression tree models to calculate forest aboveground biomass (AGB) for a mixedconifer region in eastern Oregon (USA) by using Landsatderived disturbance and recovery history (DR metrics) and lidar. the result of using DR metrics for improved predictions of $\mathrm{AGB}$ was $\mathrm{RMSE}=30.3 \mathrm{Mg} / \mathrm{ha}$ and the result of models based

\footnotetext{
${ }^{*}$ Corresponding author
} 
on single-date reflectance was RMSE $=39.6 \mathrm{Mg} / \mathrm{ha}$ (Pflugmacher, Cohen et al. 2014).

Kaili Liu et al (2017) used three modeling methods (stepwise regression (SR), support vector regression (SVR) and random forest (RF)) to estimate AGB by using Geoscience Laser Altimeter System (GLAS) data and Thematic Mapper (TM) data in the Daxing'anling Mountains in northeastern China. The result of random forest Method was been the acceptable modeling accuracy $(\mathrm{R} 2=0.95 \mathrm{RMSE}=17.73 \mathrm{Mg} / \mathrm{ha})$ and it was also been to estimate AGB by cross validation $(\mathrm{R} 2=0.71$ RMSE $=39.60 \mathrm{Mg} / \mathrm{ha}$ ) (Liu, Wang et al. 2017).

Tien Dat Pham et al (2018) used SVR model for mangrove biomass estimation from integration of ALOS-2 PALSAR-2 and Sentinel-2A data. the result of calculating AGB was with $\mathrm{RMSE}=0.187 \mathrm{Mg} / \mathrm{ha}$ and R2=0.596 (Pham, Yoshino et al. 2018).

Luodan Cao et al (2018) used Lidar and optical data for estimating AGB of forests in the upper Heihe River Basin in Northwest China by using Random Forest (RF), Support Vector Machines (SVM), Back Propagation Neural Networks (BPNN), K-Nearest Neighbor (KNN) and the Generalized Linear Mixed Model (GLMM). Using of the RF algorithm and integrated LiDAR and optical data had best result that was with R2 = 0.913, RMSE $=13.352$ t/ha (Cao, Pan et al. 2018).

This study attempted to contribute to the development of remote sensing-based predictive mapping techniques for urban forest AGB and carbon stock using freely accessible multi-source remote sensing data with a relatively high spatial resolution.

\section{PROPOSED ALGORITHM}

\subsection{Study area}

NUR is a city and located on the Caspian Sea in Mazandaran Province of northern Iran (36 $\left.34^{\prime} 32.70 " \mathrm{~N}, 52^{\circ} \quad 1^{\prime} 35.65^{\prime \prime} \mathrm{E}\right)$ (Figure 1). The study area is dominated by oriental beech (Fagus orientalis), European hornbeam (Carpinus betulus) mostly.

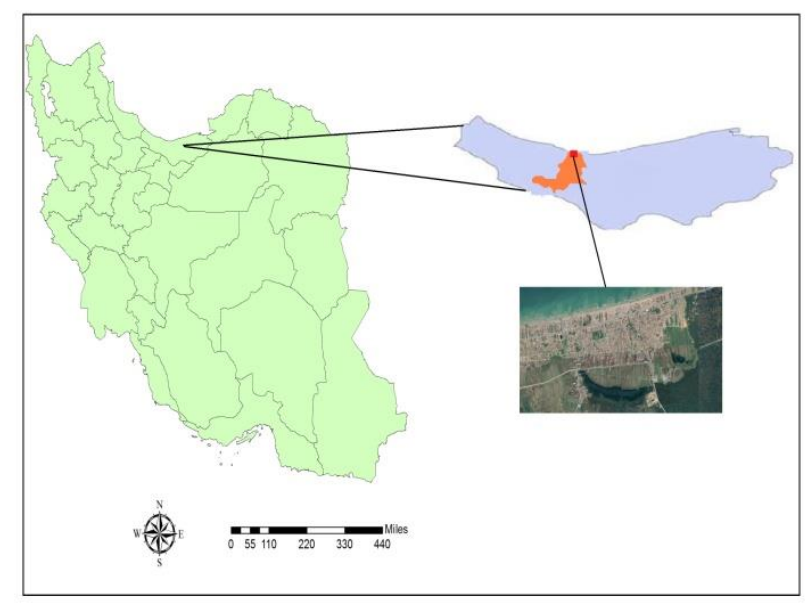

Figure 1. Location of study area in Iran. Orange colour shows Nur city. Landsat image (bottom-right map) shows study area.

\subsection{Data}

\subsubsection{Field Measurements}

A total of 65 field plots were laid out in this forest. Field measurements on the square sample plots with size of $(45 \mathrm{~m} \times$ $45 \mathrm{~m}$ ) were conducted during the summer (July) of 2014. In each plot, type of tree species and the diameter at breast height
(DBH) of all trees were measured. Trees with DBH below 7.5 $\mathrm{cm}$ were not included in the survey. The coordinates of all plot centers were measured using a Trimble real time kinematic (RTK) GPS. The AGB of each tree was calculated using the standard allometric equation (Eq.1) (Brown 1997) . AGB of each plot was calculated by summing AGB of each tree and divided by the plot.

$$
\operatorname{AGB}\left(\frac{\mathrm{Mg}}{\mathrm{hg}}\right)=\text { volume } \times \mathrm{WD}
$$

Where the volume is volume of wood $(\mathrm{m} 3 / \mathrm{ha})$ and WD is the average critical wood density (ton/m3).

\subsubsection{Remote sensing data}

\section{- Optical data}

Sentinel-2 is a satellite with Earth observation goal from the EU Copernicus Program. The satellite acquires optical imagery at high spatial resolution (from $10 \mathrm{~m}$ to $60 \mathrm{~m}$ ) over land and coastal waters and has a constellation with two twin satellites that are Sentinel-2A and Sentinel-2B. The series of Landsat satellite is achievement between NASA and USGS (United States Geological Survey).The satellite acquires spacebased images over land surface and has a medium spatialresolution. In this study, the series of Landsat images (TM, $\mathrm{ETM}+, \mathrm{OLI})$ are used.

\section{- $\quad$ Radar data}

ALOS PALSAR is the large Japanese satellite developed by JAXA (Japan Aerospace Exploration Agency) with Earth observation goal. One of the most important characteristics of the satellite is high resolution and the major observation mode is 'fine' mode.

Sentinel-1 is a satellite lead by European Space Agency (ESA) it is composed of two satellites, Sentinel-1A and Sentinel-1B, that carry a C-band SAR instrument. The satellite acquires data in all-weather and day or night.

\subsection{Biomass Estimation Approach}

To use the available satellite images and data, we must first correct the images from any errors in the process of receiving or sending images. After pre-processing of data, data indexes are extracted. After that, by the considered modelling method, the amount of biomass of plots and extraction index are communicated and total above ground biomass and carbon stock are estimated. Green spaces (urban forest) are extracted with support vector machine in time series of Landsat images. At the end, carbon stock maps in time series are simulated by using green space extraction map and total carbon stock map.

\subsubsection{Pre-processing}

Radiometric correction was worked by gray-scale pixel values Without attention of the location and geometric properties of the image components. The correction tries to improve gray scale pixel values. Radiometric corrections are used to reduce or eliminate two major errors (atmospheric errors and device errors). Images should be converted to the reference coordinate system then pixel values in different images should be compared, it is unimportant day or season of the acquired image and sun-sensor target geometry. Because of the change of viewing geometry in the path of the satellite, the reflection values for the same land cover feature are very different. 
Radar signals should be pre-processed to account for geometric distortions such as layover, foreshortening, shadow and e.g. which disturb the structure of the images and for differences in illumination conditions due to topography. The noises created by reflections from features should be removed. The noises are speckle noise and they are removed by speckle filtering. The speckle noise is main factor of disturbance of SAR image matching and reduces the radiometric quality of SAR images. In this part, the SAR and optical images are assumed that have been on the same projection and geographic coordinate system and they are co-registered at a sub pixel level (Chavez, Sides et al. 1991).

After the pre-processing, the data is also resampled to 23 meters pixel sizes. The pre-processing steps in the study are shown (Figure2).

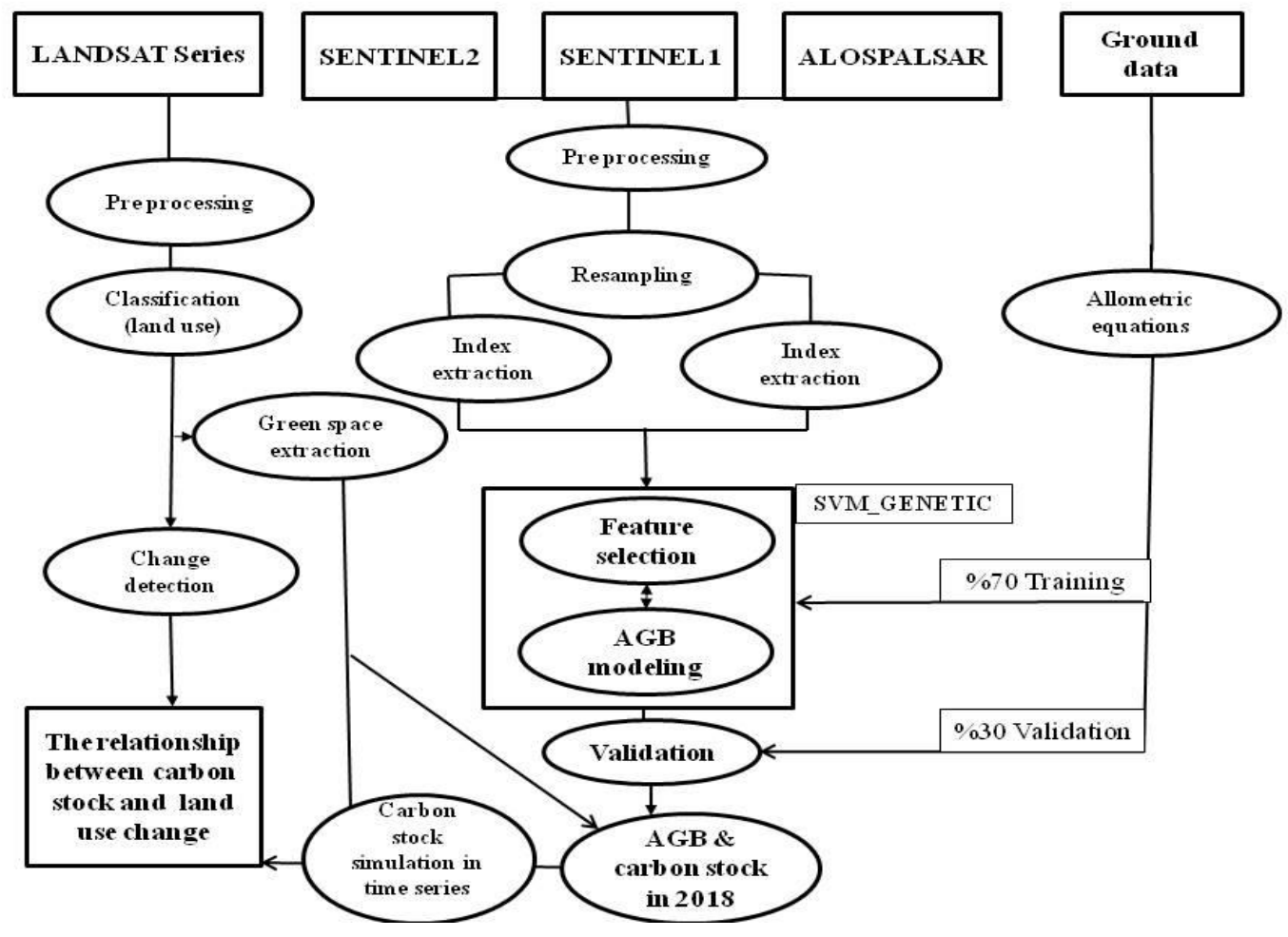

Figure 2. Flowchart of steps used for urban forest carbon stock mapping using optic and radar imagery in this study.

\subsubsection{Indexes extraction}

\section{- $\quad$ Optical index}

Vegetation Index (VI) is a spectral transformation of bands that shows vegetation properties. The index depends on absorption active radiation of vegetation. VIs correspond to biomass and are used for determine of global carbon cycle. Texture is important feature for image analysis (Haralick 1979). GLCM texture contains mean, variance, homogeneity, contrast, dissimilarity, entropy and correlation features. Principal component analysis (PCA) is used to remove redundant information in satellite data (Jolliffe and Cadima 2016). and Tasseled-Cap Transformation that is a conversion of the original bands of an image into a new set of bands with defined interpretations that are useful for vegetation mapping.

\section{- $\quad$ Radar index}

The backscatter of radar waves from the surfaces is influenced by two factors: (1) the roughness geometry and (2) the dielectric properties of the surface (Hajnsek 2001). The decomposition of Polarimetric Synthetic Aperture Radar (PolSAR) data is an important analysis step to characterize different types of backscatter and to derive higher level (beyond level 2.0) products for earth observation. Radar GLCM texture index, like optical texture index, contains mean, variance, homogeneity, contrast, dissimilarity, entropy, and correlation attributes.

\subsubsection{Green space extraction (urban forest) with Support Vector Machine (SVM)}

In this study, the classification of image objects the SVM technique (Kotsiantis, Zaharakis et al. 2007) was performed. SVM is the new classification method that isn't influenced by training data for remote sensing data with a large spectral 
dimension. This method is directly generated through an optimization process, to create a separator plane between spectral classes.

SVM separates the different classes by a hyper plane (Vapnik and Vapnik 1998, Karatzoglou, Meyer et al. 2005). This separator plane or boundary between classes is defined by using training data that has closest distance to a hyper plane and are known as support vectors. An optimum hyper plane is ascertained by using a training dataset, and its generalization ability is checked by using a validation dataset (Kavzoglu and Colkesen 2009).

\subsubsection{The GA-SVM method (AGB modelling)}

In the present study, a hybrid method, this method uses genetic algorithm to select tag, and support vector machine to predict the rest AGB. Parameters of support vector machine are optimized by particle swarm optimization algorithm. As mentioned above, this method is called as GA-SVM method with parameter optimization. Details about SVM regression can be found in literatures as in other multivariate statistical models, the performance of SVM regression depends on the combination of several parameters. In general, $\mathrm{C}$ is a regularization parameter that controls the trade off between training error and model complexity .if $\mathrm{C}$ is too large, the model will have a high penalty for no separable points and may store too many support vectors and get over fitting. If it is too small, the model may have under fitting. Parameter $\varepsilon$ controls the width of the $\varepsilon$ insensitive zone, used to fit the training data. The value of $\varepsilon$ can affect the number of the support vectors used to construct the regression function. The bigger $\varepsilon$ is, the fewer support vectors are selected. On the other hand, bigger $\varepsilon$ values result in more flat estimates. Hence, both $\mathrm{C}$ and $\varepsilon$ values affect model complexity (but in a different way). The kernel type is another important parameter. In SVM regression, radial basis function (RBF) was the most commonly used kernel function for its better generalization ability, less number of parameters, and less numerical difficulties and was used in this study. Parameter Y in RBF controls the amplitude of the RBF kernel and therefore controls the generalization ability of SVM regression. The LIBSVM package (version
2.81) was used in this study for SVM regression calculation, taking the form

$$
\mathrm{K}(\mathrm{xi}, \mathrm{x} \mathrm{j})=\exp \left(-\mathrm{Y}\|\mathrm{xi}-\mathrm{xj}\|^{2}\right) . \mathrm{Y}>0
$$

Where $\mathrm{xi}$ and $\mathrm{xj}$ are training vectors and $\mathrm{Y}$ is kernel parameter (Chang and Lin 2001).

Genetic algorithms (GA) are stochastic optimization and search method that mimics biological evolution as a problem-solving strategy. They are very flexible and attractive for optimization problems.

Given a specific problem to solve, the input to the GA is a set of potential solutions to that problem, encoded in some fashion, and a fitness function that allows each candidate to be quantitatively evaluated. Selection, mating, and mutation just mimic the natural process. For each generation, individuals are selected for reproduction according to their fitness values. Favourable individuals have a better chance to be selected for reproduction and the offspring have chance to mutate to keep diversity, while the unfavourable individuals are less likely to Survive. After each generation, whether the evolution is converged or the termination criteria are met is checked; if yes, job is done; if not, the evolution goes into next generation. After many generations, good individuals will dominate the population, and we will get solutions that are good enough for our problem.

\subsubsection{Carbon stock}

The biomass estimate is converted to carbon by the scaling factor. For tree vegetation, use the Carbon scaling factor $0.47 \mathrm{t}$ $\mathrm{C}^{-1}$ dry matter.

\section{RESULTS}

\subsection{Change detection}

In this study, four classes (water, agriculture, urban forest, urban) are regarded for classification (Figure 2). The urban forests are extracted from classification map of each considered year. The change of urban forest and manmade area (urban) acquire for each considered year. 


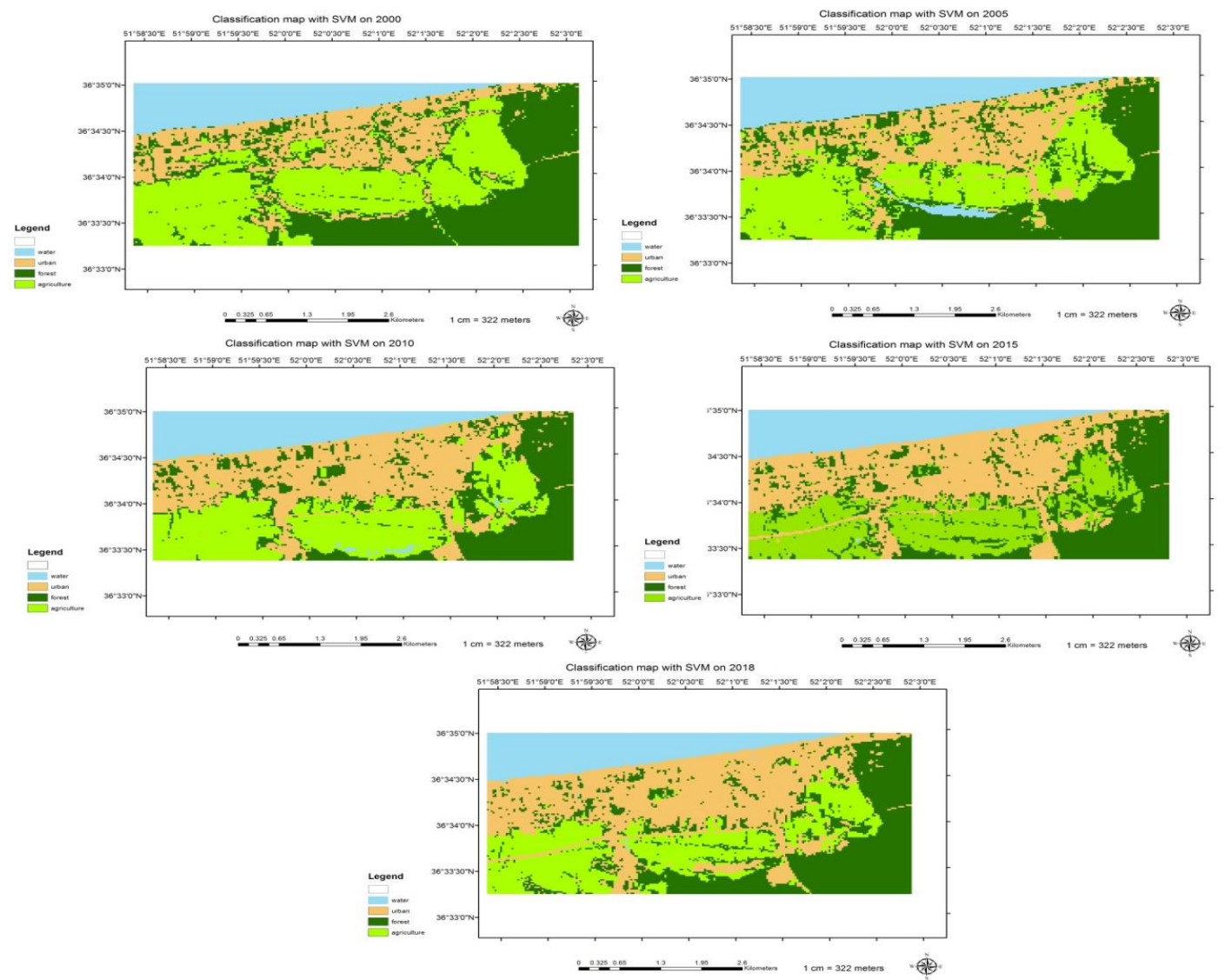

Figure 2. SVM classification map from 2000 to 2018, respectively; there are four classes; water (blue colour), urban (cream colour), agriculture (light green colour), urban forest (dark green colour).

After classification, land use maps are compared in order to obtain changed areas between 2000 and 2018.

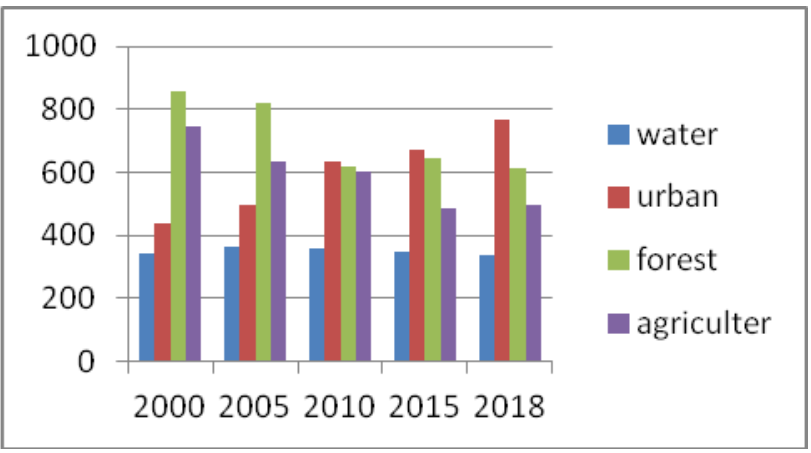

Figure 3. Bar graph of Land use area from 2000 to 2018; urban area has increased and forest area has decreased in time series.

\begin{tabular}{|c|c|c|c|c|}
\hline & water & Urban & forest & agriculture \\
\hline Water & 308.88 & 0.36 & 0.9 & 0.27 \\
\hline Urban & 13.23 & 410.85 & 182.88 & 161.1 \\
\hline forest & 0 & 12.69 & 472.68 & 128.34 \\
\hline agriculture & 0 & 8.01 & 41.49 & 445.32 \\
\hline
\end{tabular}

Table 1. Land use change from 2000 to 2018 (ha).

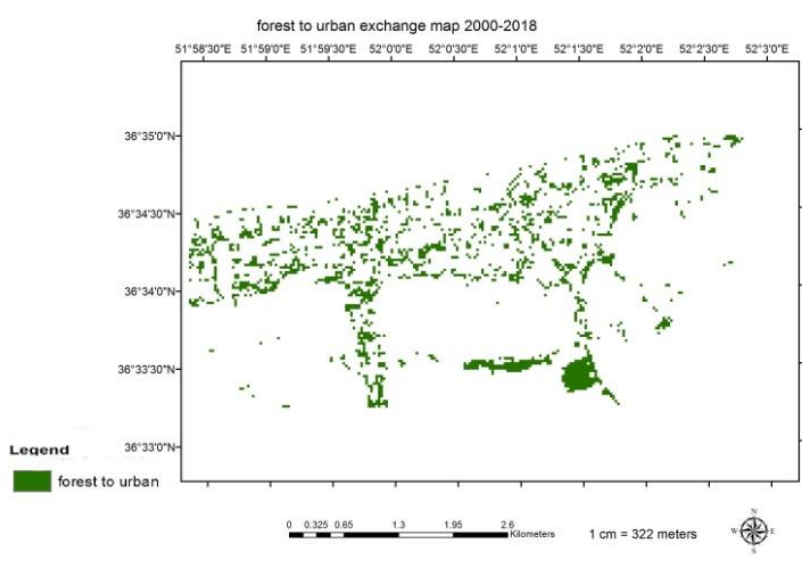

Figure 4. Forest to urban exchange map in 2000-2018; Deforestation has increased in 2018.

\subsection{Above ground biomass (AGB) in forest area}

In this part, sentinel-1, sentinel-2 and ALOS PALSAR data are used. After pre-processing and extraction of indices, the AGB and the total carbon stock map of combination of them is estimated by GA-SVM matlab code. At the end, the carbon stock of urban green space (urban forest) on 2018 is acquired by the total carbon stock map and green space extraction on 2018. 


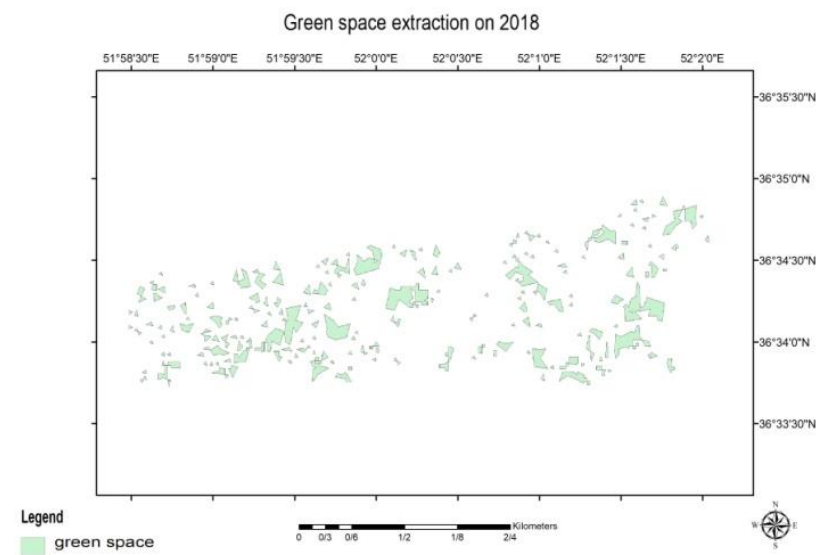

Figure5. Green space extraction in 2018

\begin{tabular}{|c|c|c|}
\hline Data & Index group & Index name \\
\hline \multirow{4}{*}{ ALOSPALSAR } & Texture & $\begin{array}{c}\text { Entropy, Entropy_shannon_I_norm, Entropy_shannon_P_norm, } \\
\text { C11_texture_dissimilarity, C11_texture_entropy, C11_texture_homogeneity }\end{array}$ \\
\cline { 2 - 3 } & Decomposition & $\begin{array}{r}\text { Combination_H1mA, Raney_Rnd, Combination_1mHA, Raney_Dbl, } \\
\text { Combination_1mH1mA, Raney_m, Combination_1mH1mA, Delta, Compact_Rsov, } \\
\text { Compact_phi, Compact_ps, Compact_p1, Compact_mv }\end{array}$ \\
\hline \multirow{5}{*}{ Sentinel-1 } & Texture & $\begin{array}{c}\text { Entropy1, C22_texture_uniformity, C22_texture_dissimilarity, C12_real1, } \\
\text { Sentinel-2 }\end{array}$ \\
\cline { 2 - 3 } & Decomposition & Raney_Rnd2, Lambda1,Delta1, Compact_tau1,Compact_Rsov, \\
& & Compact_ps1,Compact_p11, Compact_mv1,Compact_121, Compact_111, \\
& Combination_HA1, Combination_H1mA1, Compact_alpha_s1
\end{tabular}

Table 2. The best selection indexes of the data for biomass estimation in GA-SVM.

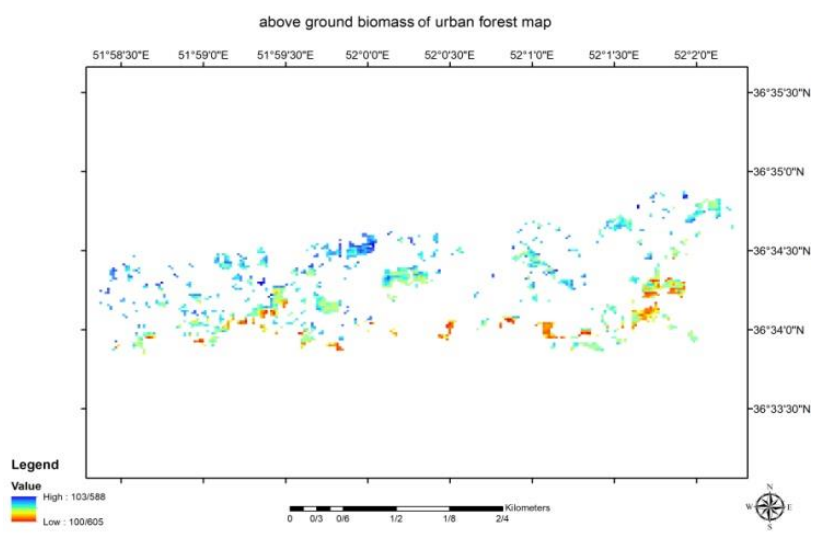

Figure 6. Above ground biomass of urban forest map in 2018

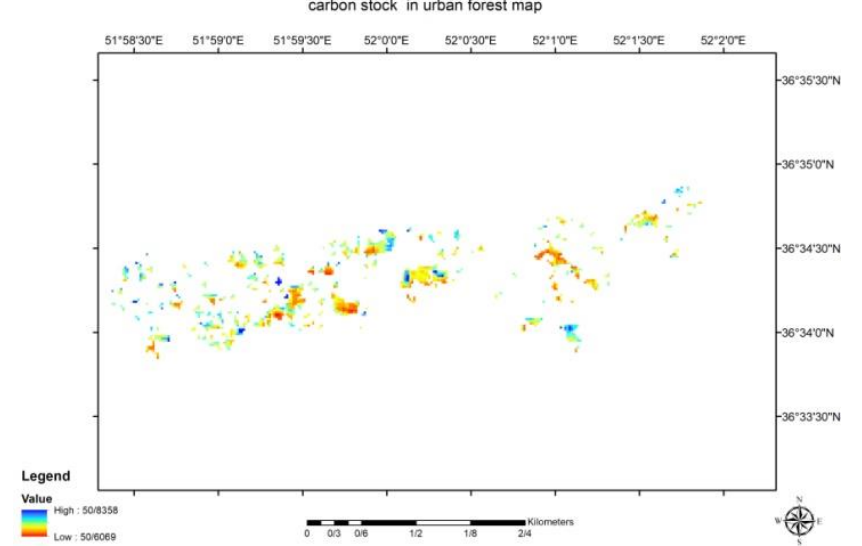

Figure 7. Carbon stock of urban forest in 2018 


\subsection{Simulate of the carbon stock in time series}

At the end part, the carbon stock map on 2000, 2005, 2010 and 2015 are simulated by the carbon stock of green space estimated map on 2018 and the green space extraction of each year with Kiriging interpolation (Stein 2012).

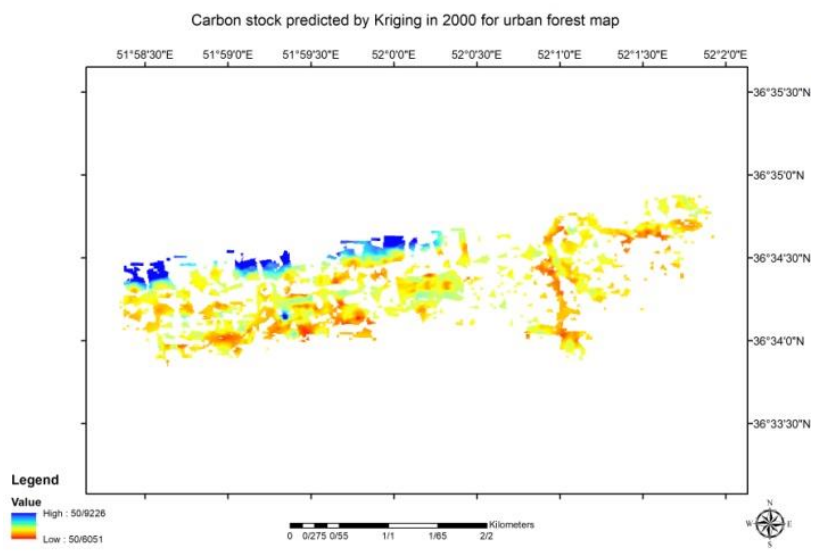

Figure 8. Carbon stock of urban forest in 2000

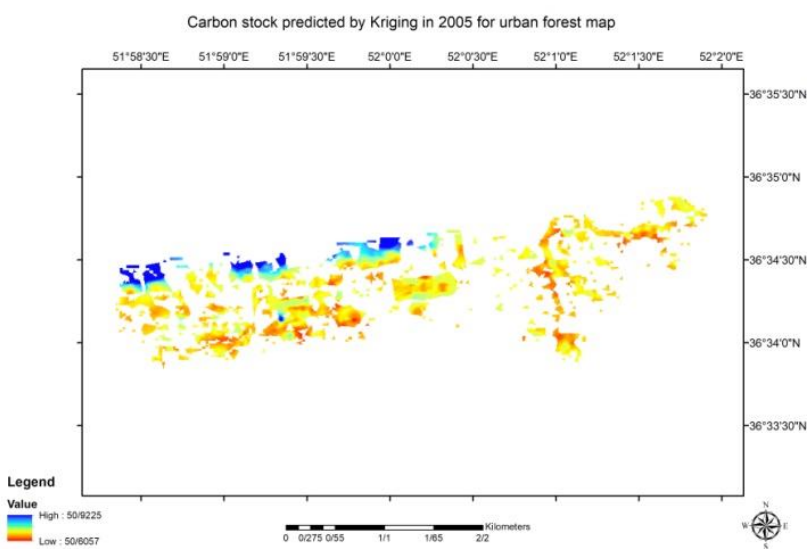

Figure 9. Carbon stock of urban forest in 2005

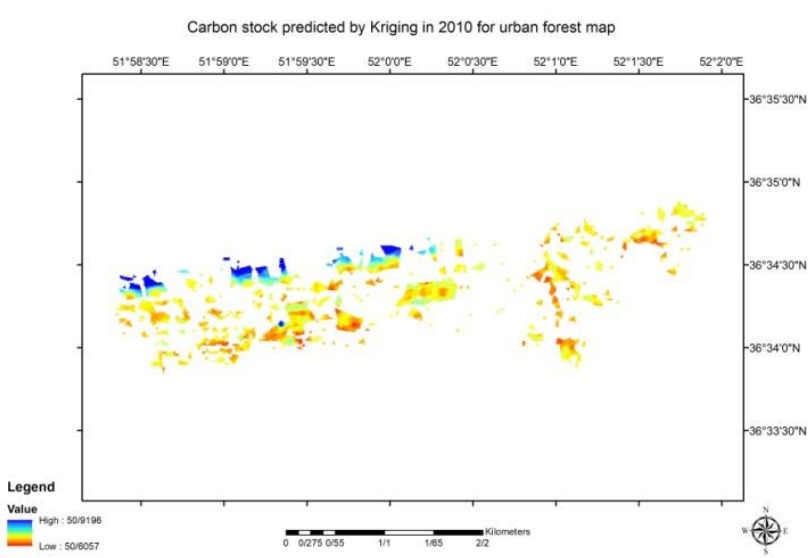

Figure 10. Carbon stock of urban forest in 2010

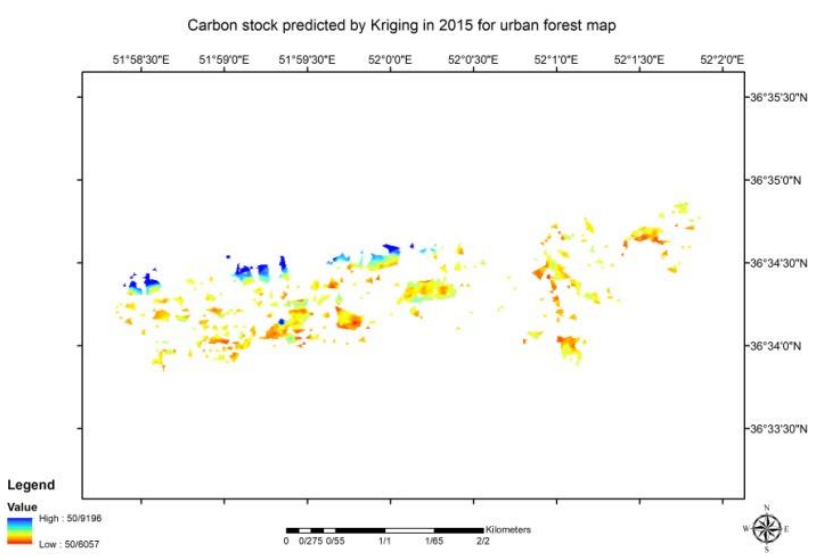

Figure 11. Carbon stock of urban forest in 2015

\begin{tabular}{|c|c|c|c|c|}
\hline 2018 & 2015 & 2010 & 2005 & 2000 \\
\hline 469.05193 & 670.6051 & 897.13379 & 1302.45 & 1671.4052 \\
\hline
\end{tabular}

Table 3. The amount of Carbon stock (ton/ha)

Carbon cycle has decreased with the increase in deforestation. The lowest rate of carbon stock is observed in 2018. 182.88 ha of forest area in 2000 have been converted into urban area in 2018 and 1202.35327 ton/ha of carbon have decreased since 2000 to 2018 .

\section{CONCLUSION}

Literature demonstrates that there is a decline in the number of studies using conventional methods to estimate AGB, compared to remote sensing methods. Conventional methods, although accurate, are time-consuming, too costly and practically impossible to apply on a broader scale. Although active sensors, such as Lidar and radar, provide higher and more reliable AGB estimates than coarse multispectral data, they are still not operational in the environments. The growth rate of cities has a direct relation with the reduction of biomass in the city.

\section{REFRENCE}

Brown, S. (1997). Estimating biomass and biomass change of tropical forests: a primer, Food \& Agriculture Org.

Cao, L., J. Pan, R. Li, J. Li and Z. Li (2018). "Integrating airborne LiDAR and optical data to estimate forest aboveground biomass in arid and semi-arid regions of China." $\underline{\text { Remote }}$ Sensing 10(4): 532.

Chang, C.-C. and C.-J. Lin (2001). "Training v-support vector classifiers: theory and algorithms." Neural computation 13(9): 2119-2147.

Chavez, P., S. C. Sides and J. A. Anderson (1991). "Comparison of three different methods to merge multiresolution and multispectral data- Landsat TM and SPOT panchromatic." Photogrammetric Engineering and remote sensing 57(3): 295-303.

Fassnacht, F., F. Hartig, H. Latifi, C. Berger, J. Hernández, P. Corvalán and B. Koch (2014). "Importance of sample size, data type and prediction method for remote sensing-based estimations of aboveground forest biomass." Remote Sensing of Environment 154: 102-114.

Hajnsek, I. (2001). "Inversion of surface parameters using polarimetric SAR." 
Haralick, R. M. (1979). "Statistical and structural approaches to texture." Proceedings of the IEEE 67(5): 786-804.

Jolliffe, I. T. and J. Cadima (2016). "Principal component analysis: a review and recent developments." Philosophical Transactions of the Royal Society A: Mathematical, Physical and Engineering Sciences 374(2065): 20150202.

Karatzoglou, A., D. Meyer and K. Hornik (2005). "Support vector machines in R."

Kavzoglu, T. and I. Colkesen (2009). "A kernel functions analysis for support vector machines for land cover classification." International Journal of Applied Earth Observation and Geoinformation 11(5): 352-359.

Kotsiantis, S. B., I. Zaharakis and P. Pintelas (2007). "Supervised machine learning: A review of classification techniques." Emerging artificial intelligence applications in computer engineering 160: 3-24.

Kwak, D.-A., W.-K. Lee, H.-K. Cho, S.-H. Lee, Y. Son, M. Kafatos and S.-R. Kim (2010). "Estimating stem volume and biomass of Pinus koraiensis using LiDAR data." Journal of plant research 123(4): 421-432.

Laurin, G. V., Q. Chen, J. A. Lindsell, D. A. Coomes, F. Del Frate, L. Guerriero, F. Pirotti and R. Valentini (2014). "Above ground biomass estimation in an African tropical forest with lidar and hyperspectral data." ISPRS Journal of Photogrammetry and Remote Sensing 89: 49-58.

Liu, K., J. Wang, W. Zeng and J. Song (2017). "Comparison and evaluation of three methods for estimating forest above ground biomass using TM and GLAS data." Remote Sensing 9(4): 341 .

Lu, D. (2006). "The potential and challenge of remote sensing-based biomass estimation." International journal of remote sensing 27(7): 1297-1328.

Pataki, D. E., M. M. Carreiro, J. Cherrier, N. E. Grulke, V. Jennings, S. Pincetl, R. V. Pouyat, T. H. Whitlow and W. C. Zipperer (2011). "Coupling biogeochemical cycles in urban environments: ecosystem services, green solutions, and misconceptions." Frontiers in Ecology and the Environment 9(1): 27-36.

Pflugmacher, D., W. B. Cohen, R. E. Kennedy and Z. Yang (2014). "Using Landsat-derived disturbance and recovery history and lidar to map forest biomass dynamics." Remote Sensing of Environment 151: 124-137.

Pham, T. D., K. Yoshino, N. N. Le and D. T. Bui (2018). "Estimating aboveground biomass of a mangrove plantation on the Northern coast of Vietnam using machine learning techniques with an integration of ALOS-2 PALSAR-2 and Sentinel-2A data." International journal of remote sensing 39(22): 7761-7788.

Rao, P., L. R. Hutyra, S. M. Raciti and A. C. Finzi (2013). "Field and remotely sensed measures of soil and vegetation carbon and nitrogen across an urbanization gradient in the Boston metropolitan area." Urban Ecosystems 16(3): 593-616.

Saatchi, S. S., N. L. Harris, S. Brown, M. Lefsky, E. T. Mitchard, W. Salas, B. R. Zutta, W. Buermann, S. L. Lewis and S. Hagen (2011). "Benchmark map of forest carbon stocks in tropical regions across three continents." Proceedings of the national academy of sciences 108(24): 9899-9904.

Seto, K. C., S. Dhakal, A. Bigio, H. Blanco, G. C. Delgado, D. Dewar, L. Huang, A. Inaba, A. Kansal and S. Lwasa (2014). "Human settlements, infrastructure and spatial planning."

Stein, M. L. (2012). Interpolation of spatial data: some theory for kriging, Springer Science \& Business Media.

UN. (2015). "World Urbanization Prospects: The 2014 Revision."
Vapnik, V. and V. Vapnik (1998). "Statistical learning theory Wiley." New York: 156-160. 